\title{
A Semiempirical Approach to the Intra-Phycocyanin and Inter-Phycocyanin Fluorescence Resonance Energy-Transfer Pathways in Phycobilisomes
}

\author{
ADELIO R. MATAMALA, ${ }^{1}$ DANIEL E. ALMONACID, ${ }^{1 *}$ MAXIMILIANO F. FIGUEROA, ${ }^{2}$ \\ JOSÉ MARTÍNEZ-OYANEDEL, ${ }^{2}$ MARTA C. BUNSTER ${ }^{2}$ \\ ${ }^{I}$ Facultad de Ciencias Químicas, Universidad de Concepción, Casilla 160-C, Edmundo Larenas \\ 129, Concepción, Chile \\ ${ }^{2}$ Laboratorio de Biofísica Molecular, Grupo de Biología Estructural, Departamento de \\ Bioquímica y Biología Molecular, Facultad de Ciencias Biológicas, Universidad de Concepción, \\ Concepción, Chile
}

Received 18 July 2006; Revised 20 October 2006; Accepted 23 October 2006

DOI 10.1002/jcc.20628

Published online 13 February 2007 in Wiley InterScience (www.interscience.wiley.com).

\begin{abstract}
A semiempirical methodology to model the intra-phycocyanin and inter-phycocyanin fluorescence resonance energy-transfer (FRET) pathways in the rods of the phycobilisomes (PBSs) from Fremyella diplosiphon is presented. Using the Förster formulation of FRET and combining experimental data and PM3 calculation of the dipole moments of the aromatic portions of the chromophores, transfer constants between pairs of chromophores in the phycocyanin (PC) structure were obtained. Protein docking of two PC hexamers was used to predict the optimal distance and axial rotation angle for the staked PCs in the PBSs' rods. Using the distance obtained by the docking process, transfer constants between pairs of chromophores belonging to different PC hexamers were calculated as a function of the angle of rotation. We show that six preferential FRET pathways within the PC hexameric ring and 15 pathways between hexamers exist, with transfer constants consistent with experimental results. Protein docking predicted the quaternary structure for PCs in rods with inter-phycocyanin distance of $55.6 \AA$ and rotation angle of $20.5^{\circ}$. The inter-phycocyanin FRET constant between chromophores at positions $\beta_{155}$ is maximized at the rotation angle predicted by docking revealing the crucial role of this specific inter-phycocyanin channel in defining the complete set of FRET pathways in the system.
\end{abstract}

(C) 2007 Wiley Periodicals, Inc. J Comput Chem 28: 1200-1207, 2007

Key words: semiempirical; dipole moment; FRET; phycocyanin; protein docking

\section{Introduction}

Cyanobacteria and eukaryotic red algae contain elaborate light harvesting antenna complexes in addition to chlorophyll molecules. These antenna systems absorb the incident sunlight in portions of the visible spectrum poorly utilized by chlorophyll and convey the energy to the photosynthetic reaction centers through a fluorescence resonance energy-transfer (FRET) mechanism. ${ }^{1-6}$ Each antenna system is made up of chromophore-containing proteins called phycobiliproteins (PBPs). The PBPs are arranged in subcellular structures called phycobilisomes (PBSs) at the surface of the thylakoid membranes. ${ }^{7,8}$ The PBSs allow the chromophores to adopt an appropriate geometry for the capture of light and the transfer of energy with an impressively high efficiency. ${ }^{9,10}$ PBSs from different species differ in their polypeptide composition. However, they share a general common morphology that consists of a core of face-to-face cylinders formed by staked trimeric discs of PBPs and several rods that radiate from the core, composed of back-to-back stacked hexameric discs of PBPs. ${ }^{11,12}$ Each PBP consists of $\alpha$ and $\beta$ polypeptide subunits to which one or more linear tetrapyrrole chromophores are covalently attached. These $\alpha$ and $\beta$ subunits associate to form $\alpha \beta$ monomers and six of these monomers form the $(\alpha \beta)_{6}$ hexameric units, which pile up to form the PBS rods. Phycocyanin $(\mathrm{PC})$ is one of the PBPs that is present in the rods of the PBSs. The chromophore in PC, phycocyanobilin, is attached by

*Present address: Unilever Centre for Molecular Science Informatics, Department of Chemistry, University of Cambridge, Lensfield Road, Cambridge, CB2 1EW, UK.

Correspondence to: A. R. Matamala; e-mail: amatamal@udec.cl

Contract/grant sponsor: Universidad de Concepción (Chile); contract/ grant numbers: DIUC 205.037.002 
cysteinyl thioether linkages at positions $\alpha_{84}, \beta_{84}$, and $\beta_{155}$ in the $\alpha$ and $\beta$ subunits.

The supramolecular structures of PBSs have been determined for many organisms including Synechocystis, ${ }^{13,14}$ Synechococcus, ${ }^{15-18}$ and Anabaena. ${ }^{11,19}$ However, the study of light transfer in PBSs remains a challenging task from both an experimental and theoretical point of view. Since the first three-dimensional high resolution structure of a $\mathrm{PC}$ was reported, ${ }^{20}$ considerable efforts have been devoted to modeling the energy-transfer processes among chromophores within and between $(\alpha \beta)_{6}$ hexameric rings. ${ }^{21-26}$ Förster $^{2-4}$ introduced a very basic formulation for the energy-transfer mechanism between two chromophores, the socalled FRET mechanism. A fluorescent molecule (the donor) is excited, after which its energy is nonradiatively transferred to a second molecule (the acceptor), which becomes excited while the donor molecule returns to the electronic ground state. This process occurs under resonance conditions, i.e., under conservation of the total energy. Part of the electronic energy may be transformed into vibrational energy resulting in small inefficiencies due to thermal dissipation. The original theory of FRET was derived under strong assumptions: long-range dipole-dipole transfer regime and single-chromophore interactions. Dexter ${ }^{27}$ improved the formulation of FRET to include higher multipole and exchange interactions. Recent experimental and theoretical developments have suggested the necessity of a theory of FRET considering multichromophoric processes. ${ }^{28-32}$ However, the original Förster's formulation of FRET has been confirmed to be an excellent approximation to model the light transfer processes occurring in PBPs. ${ }^{33-34}$

In his formulation, Förster ${ }^{2-4}$ defined an energy-transfer constant characterized by the spectroscopic properties and the interaction geometry of the participating chromophores. The latter is known as the geometric factor and includes the distance between the centers of mass for each pair of chromophores and the dipole-dipole orientation coefficient and is the most influential term in the Förster equation. ${ }^{35}$ Distances between chromophores can be extracted easily from high resolution structures of PBPs, however dipole-dipole orientation coefficients need the determination of the direction of the dipole moment of the chromophores. To obtain the direction of the dipole moments, Schirmer and colleagues, as a first approximation, fitted a line to the conjugated part of the chromophores by least squares methods. ${ }^{36} \mathrm{~A}$ more advanced determination of the dipole moment of chromophores in PC has been carried out by Scharnagl and Schneider. ${ }^{35}$ They used the PPP method and considered the whole chromophores and their surrounding amino acid residues as a "super molecule" and concluded that the direction of the dipole moments is essentially parallel to the long axis of the chromophores. Despite FRET formulation being strongly dependant on the dipole moments of the interacting chromophores, all studies of FRET in PBSs up to this date have been carried out using dipole moments determined as fitted lines to either the conjugated part or the long axis of the chromophores, approximations that are not well justified.

Here, we present a semiempirical methodology to determine the direction of the dipole moment of the chromophores in $\mathrm{PC}$ and model the intra-PC and inter-PC FRET pathways in the PBSs from Fremyella diplosiphon. ${ }^{21}$ In section one, we present
PM3 semiempirical dipole moments for the chromophores in PC and use Förster's formulation of FRET to determine intra-phycocyanin preferential light transfer pathways. In section two, a protein docking of two PC hexamers is presented to determine the optimal distance and axial rotation angle for the staked PCs in the PBSs' rods. In section three, using the distance determined by docking, inter-phycocyanin FRET pathways were studied as a function of the inter-phycocyanin rotation angle.

\section{Methods}

According to the Förster formulation of FRET, ${ }^{2-4,33}$ the frequency of energy transfer events between a donor-acceptor pair of chromophores is defined by the so-called transfer rate constant, $K_{\mathrm{DA}}$, given by:

$$
K_{\mathrm{DA}}=C G S I
$$

where $C$ is a collection of constants, $G$ is the geometric factor, $S$ includes the spectroscopic properties of the interacting chromophores, and $I$ is the overlap integral between the emission and absorption fluorescence spectra of the donor and acceptor chromophores, respectively.

In eq. (1), the constant $C$ includes the Avogadro number, $N_{\mathrm{A}}$, and the refractive index of the surrounding medium, $n$,

$$
C=\frac{9 \ln 10}{128 \pi^{5} N_{\mathrm{A}} n^{4}}
$$

The geometric factor $G$,

$$
G=\frac{\kappa_{\mathrm{DA}}^{2}}{R_{\mathrm{DA}}^{6}},
$$

includes the distance between the centers of mass for each pair of chromophores, RDA, and the dipole-dipole orientation coefficient:

$$
\kappa_{\mathrm{DA}}=\hat{\mu}_{\mathrm{D}} \hat{\mu}_{\mathrm{A}}-3\left(\hat{\mu}_{\mathrm{D}} \hat{r}_{\mathrm{DA}}\right)\left(\hat{\mu}_{\mathrm{A}} \hat{r}_{\mathrm{DA}}\right),
$$

where $\hat{\mu}_{\mathrm{D}}$ and $\hat{\mu}_{\mathrm{A}}$ are unit vectors in the direction of the dipole of the donor and acceptor chromophores, respectively, and $\hat{r}_{\mathrm{DA}}$ is the unit vector along the line that passes through both donor and acceptor centers of mass. The term $S$ is given by:

$$
S=\frac{\Phi_{\mathrm{D}}}{\tau_{\mathrm{D}}} \varepsilon_{\mathrm{A}},
$$

where $\Phi_{\mathrm{D}}$ and $\tau_{\mathrm{D}}$ are the quantum efficiency and the life-time of the fluorescence process of the donor chromophore, respectively, and $\varepsilon_{\mathrm{A}}$ is the molar extinction coefficient of the acceptor chromophore. Finally, the term $I$ is given by the following integral:

$$
I=\int_{0}^{\infty} F_{\mathrm{D}}(\lambda) A_{\mathrm{A}}(\lambda) \lambda^{4} d \lambda,
$$

where $F_{\mathrm{D}}(\lambda)$ and $A_{\mathrm{A}}(\lambda)$ are the normalized fluorescence spectrum of the donor and absorption spectrum of the acceptor chromophores, respectively.

The atomic coordinates of the constitutive phycocyanin (PC) from Fremyella diplosiphon were extracted under accession code $1 \mathrm{cpc}$ from the Protein Data Bank. This structure was 
Table 1. Quantum Efficiency, $\Phi$, Fluorescence Life time, $\tau$, and Molar Extinction Coefficient, $\varepsilon$, for the Chromophores.

\begin{tabular}{lccc}
\hline Chromophore & $\Phi$ & $\tau(n s)$ & $\begin{array}{c}\varepsilon \times 10^{-8} \\
\left(\mathrm{~cm}^{2} \mathrm{~mol}^{-1}\right)\end{array}$ \\
\hline$\alpha_{84}$ & 0.23 & 1.50 & 1.15 \\
$\beta_{84}$ & 0.19 & 1.45 & 0.70 \\
$\beta_{155}$ & 0.25 & 0.93 & 1.12 \\
\hline
\end{tabular}

Data were taken from ref. 33.

obtained by Duerring et al. ${ }^{21}$ from X-ray data with an R-factor of $18.1 \%$ and resolution of $1.66 \AA$. Each unit cell consists of two $\alpha \beta$ heterodimers, which each contain three tetrapyrrole chromophores (phycocyanobilins) at positions $\alpha_{84}, \beta_{84}$, and $\beta_{155}$. The $(\alpha \beta)_{6}$ hexameric ring (containing 18 chromophore molecules) was obtained by the application of the R3-symmetry generator. The labeling of the subunits is in accordance with the symmetry operation as suggested in ref. 36 .

The dipole moment of the aromatic portion of each chromophore was calculated as a single point for their conformation in the PC hexamer using the PM3 semiempirical method ${ }^{37}$ implemented in Gaussian $98 .^{38}$ The correct hybridization of the atoms in the aromatic portions was maintained by substituting the connected non-hydrogen atoms with hydrogen. The distance between the centers of mass for each pair of chromophores was calculated after transforming the atomic coordinates from the standard system of reference (Gaussian output) to the actual coordinates in the PC structure.

Values for the parameters contained in the $S$ and $I$ terms were taken from ref. 33 and are shown in Tables 1 and 2. These are experimental values for the chromophores in PC from Synechococcus sp. PCC 7002 and are assumed to be valid for the set of chromophores in PC from Fremyella diplosiphon studied in the present work based on the structural similarity of the chromophores. ${ }^{21}$ In addition, both PCs share the same residues that modulate the spectral properties of the chromophores through Coulombic and van der Waals' interactions. ${ }^{21,39}$ The refractive index, $n$, was set to the value 1.567 on the basis of experimental results. $^{40,41}$

To define the geometrical arrangement adopted by the stacked PCs in the rods of physiological phycobilisomes (PBSs), two PC hexamers were docked using the program BiGGER ${ }^{42,43}$

Table 2. Fluorescence Overlap Integral, I, Between Donor-Acceptor Pair of Chromophores.

\begin{tabular}{lccc}
\hline & \multicolumn{3}{c}{ Acceptor } \\
\cline { 2 - 4 } Donor & $\alpha_{84}$ & $\beta_{84}$ & $\beta_{155}$ \\
\hline$\alpha_{84}$ & 4.50 & 7.49 & 2.16 \\
$\beta_{84}$ & 3.47 & 6.96 & 1.25 \\
$\beta_{155}$ & 7.81 & 9.97 & 5.60 \\
\hline
\end{tabular}

Data were taken from ref. 33. with an angular step of $15^{\circ}$. The 5000 models generated are presented ranked according to five different parameters: geometric complementarity, side chain contacts, electrostatic energy, solvation energy, and global score. The latter is a value defined by BiGGER that combines the former four parameters to indicate the likeliness of a model to represent a near-native solution and it ranges from 0 to 1 . To have a representative sample of the best putative models, the 20 top scored models for each of the five interaction parameters calculated by the software were selected. Each of the selected models was then visually inspected to determine whether they did or did not show the back-to-back type of interaction reported for PCs in rods as seen by electronic microscopy, ${ }^{11,18,44}$ i.e., PC hexamers positioned one on top of another maintaining an axial symmetry. Displacement along the symmetry axis passing through both centers of the PC rings and rotations around the same axis were considered to cluster the putative models. The inter-phycocyanin distance of the models in the best scored cluster was then used for investigating inter-phycocyanin FRET constants as a function of the angle of rotation. Because of the threefold symmetry of the system, a rotation range of $120^{\circ}$ was studied.

In this work, to describe energy transfer between chromophores, we use the term pathway. This term refers to single or multiple consecutive steps of energy transfer between pair of chromophores where the Förster constants are the highest for the system. Preferential pathways refer to those pathways that are compatible with empirical information and also with a global model for energy transfer.

\section{Results and Discussions}

\section{Intra-Phycocyanin Preferential Light Transfer Pathways}

Table 3 shows the mass centers and PM3 dipole moments obtained for the aromatic portions of the phycocyanobilins in the unit cell of PC from Fremyella diplosiphon. Using the data in Tables 1-3, FRET constants were determined for all possible acceptor-donor pairs within a hexamer of PC. Light transfer between chromophores $\alpha_{84}^{4}-\beta_{84}^{6}$ (and their symmetry equivalents $\alpha_{84}^{5}-\beta_{84}^{4}$ and $\left.\alpha_{84}^{6}-\beta_{84}^{5}\right)$ are the fastest, taking 5.96 ps on average. These are followed by transfer steps between chromophores $\alpha_{84}^{1}$ $-\beta_{84}^{2}$ (and their symmetry equivalents $\alpha_{84}^{2}-\beta_{84}^{3}$ and $\alpha_{84}^{3}-\beta_{84}^{1}$ ) with an average time of $9.86 \mathrm{ps}$ and between chromophores $\alpha_{84}^{1}-\alpha_{84}^{4}$ (and their symmetry equivalents $\alpha_{84}^{2}-\alpha_{84}^{5}$ and $\alpha_{84}^{3}-\alpha_{84}^{6}$ ) with an

Table 3. Mass Center and PM3 Dipole Moment for the Aromatic Portions of the Chromophores in the Unit Cell of Phycocyanin From Fremyella diplosiphon (pdb Accession Code 1cpc).

\begin{tabular}{lll}
\hline Chromophore & \multicolumn{1}{c}{ Mass center } & PM3 dipole moment \\
\hline$\alpha_{84}^{1}$ & {$[29.26,-26.34,12.23]$} & {$[0.81,-0.67,-0.69]$} \\
$\beta_{84}^{1}$ & {$[-17.56,-10.77,17.32]$} & {$[-0.56,-0.11,0.96]$} \\
$\beta_{155}^{1}$ & {$[-15.02,-43.93,8.43]$} & {$[0.42,0.20,1.85]$} \\
$\alpha_{84}^{6}$ & {$[-33.52,-20.71,-12.39]$} & {$[-1.84,-0.74,0.94]$} \\
$\beta_{84}^{6}$ & {$[15.36,-13.83,-17.49]$} & {$[1.02,-0.35,-1.14]$} \\
$\beta_{155}^{6}$ & {$[7.22,-46.12,-8.34]$} & {$[-0.91,0.43,-2.18]$}
\end{tabular}


Table 4. Highest Intra-Phycocyanin Fluorescence Resonance Energy Transfer Constants.

\begin{tabular}{lcccc}
\hline Pair & $\begin{array}{c}\text { Distance } \\
(\AA)\end{array}$ & $\kappa$ & $K_{\mathrm{DA}}^{-1}(\mathrm{ps})$ & $K_{\mathrm{AD}}^{-1}(\mathrm{ps})$ \\
\hline$\alpha_{84}^{4}-\beta_{84}^{6}$ & 20.6 & -1.04 & 5.98 & 9.20 \\
$\alpha_{84}^{5}-\beta_{84}^{4}$ & 20.6 & -1.04 & 5.94 & 9.15 \\
$\alpha_{84}^{6}-\beta_{84}^{5}$ & 20.6 & -1.04 & 5.96 & 9.18 \\
$\alpha_{84}^{1}-\beta_{84}^{2}$ & 20.6 & -0.802 & 10.0 & 15.4 \\
$\alpha_{84}^{2}-\beta_{84}^{3}$ & 20.6 & -0.810 & 9.86 & 15.2 \\
$\alpha_{84}^{3}-\beta_{84}^{1}$ & 20.6 & -0.815 & 9.73 & 15.0 \\
$\alpha_{84}^{1}-\alpha_{84}^{4}$ & 26.3 & 1.13 & 22.6 & 22.6 \\
$\alpha_{84}^{2}-\alpha_{84}^{5}$ & 26.3 & 1.13 & 22.6 & 22.6 \\
$\alpha_{84}^{3}-\alpha_{84}^{6}$ & 26.3 & 1.13 & 22.6 & 22.6 \\
$\beta_{155}^{1}-\beta_{155}^{6}$ & 27.9 & -0.658 & 44.7 & 44.7 \\
$\beta_{155}^{2}-\beta_{155}^{4}$ & 27.9 & -0.658 & 44.7 & 44.7 \\
$\beta_{155}^{3}-\beta_{155}^{5}$ & 27.9 & -0.660 & 44.5 & 44.5 \\
\hline
\end{tabular}

For each of the interacting pair of chromophores, distances between their mass centers and orientation coefficients $(\kappa)$ between their dipole moments are shown. The reciprocal of the forward $\left(1 / K_{\mathrm{DA}}\right)$ and backward $\left(1 / K_{\mathrm{AD}}\right)$ Förster constant in picosecond units are also included. The latter are estimates of the time a photon takes to be transferred from the donor chromophore (D) to the acceptor chromophore (A) and vice versa. Because the PC hexamer under study was created by applying symmetry operators to the structure of a dimer, transfer constants between pair of symmetry equivalent chromophores (e.g., $\alpha_{84}^{4}-\beta_{84}^{6}, \alpha_{84}^{5}-\beta_{84}^{4}$, and $\alpha_{84}^{6}-$ $\left.\beta_{84}^{5}\right)$ are very similar.

average time of $22.6 \mathrm{ps}$. All the above-mentioned transfer steps can be joined to define three internal pathways: $\left[\beta_{84}^{1}-\alpha_{84}^{3}-\alpha_{84}^{6}-\right.$ $\left.\beta_{84}^{5}\right],\left[\beta_{84}^{2}-\alpha_{84}^{1}-\alpha_{84}^{4}-\beta_{84}^{6}\right]$, and $\left[\beta_{84}^{3}-\alpha_{84}^{2}-\alpha_{84}^{5}-\beta_{84}^{4}\right]$. These pathways connect chromophores $\beta_{84}$ from different trimers in 43.6 ps. $\beta_{155}-\beta_{155}$ light transfer steps take $44.6 \mathrm{ps}$ on average and on their own define three external pathways: $\left[\begin{array}{lll}\beta_{155}^{1} & -\beta_{155}^{6}\end{array}\right]$, $\left[\beta_{155}^{2} \beta_{15}^{4}\right.$, and $\left[\beta_{155}^{3}-\beta_{155}^{5}\right]$ connecting chromophores $\beta_{155}$ from different trimers. Photons absorbed by any chromophore in the upper trimer of PC will be transferred to chromophores in the lower trimer of the PC hexamer by one of these pathways. We refer to these pathways as preferential pathways and summarize them in Table 4 and Figure 1.

The model for light transfer shown in Table 4 is striking in that it only uses the 12 transfer steps with the shortest transfer times out of the 153 possible pairs of chromophores inside a PC hexamer. Six preferential pathways allow defining short intraphycocyanin transfer times, because of a small number of quick steps per pathway (between one and three). This model is compatible with the short time and high quantum efficiency observed for energy transfer in PBPs and PBSs. ${ }^{9,10}$ If we were to consider the next shortest transfers, i.e., those between chromophores $\beta_{84}^{1}$ $-\beta_{84}^{5}, \beta_{84}^{2}-\beta_{84}^{6}$, and $\beta_{84}^{3}-\beta_{84}^{4}$, the model does not gain any extra functionality as a pathway connecting chromophores $\beta_{84}$ from different trimers already exists. Also, these steps are too slow (101 ps) to be of importance in the energy transfer of a phycobilisome in vivo.

An analysis of the intra-phycocyanin light transfer pathways in rods of Fremyella diplosiphon has been previously published. ${ }^{21}$ In their work, Duerring et al. approximated the dipole moments to the line fitted to the conjugated portions of the chro- mophores, and reported dipole moments in the opposite direction, which differ on average by $150^{\circ}$ to those reported in this study. Transfer constants of $6.24,38.3$, and 108 ps for pairs $\alpha_{84}-\beta_{84}, \alpha_{84}-\alpha_{84}$, and $\beta_{155}-\beta_{155}$, respectively, were reported on the basis of their calculations. The differences between the results previously obtained and those presented here are principally because of the different determination of the dipole moments of the chromophores. Because the $\pi-\pi^{*}$ electronic transitions, for quantum states of the delocalized electrons belonging to each chromophore are involved in the energy trans-

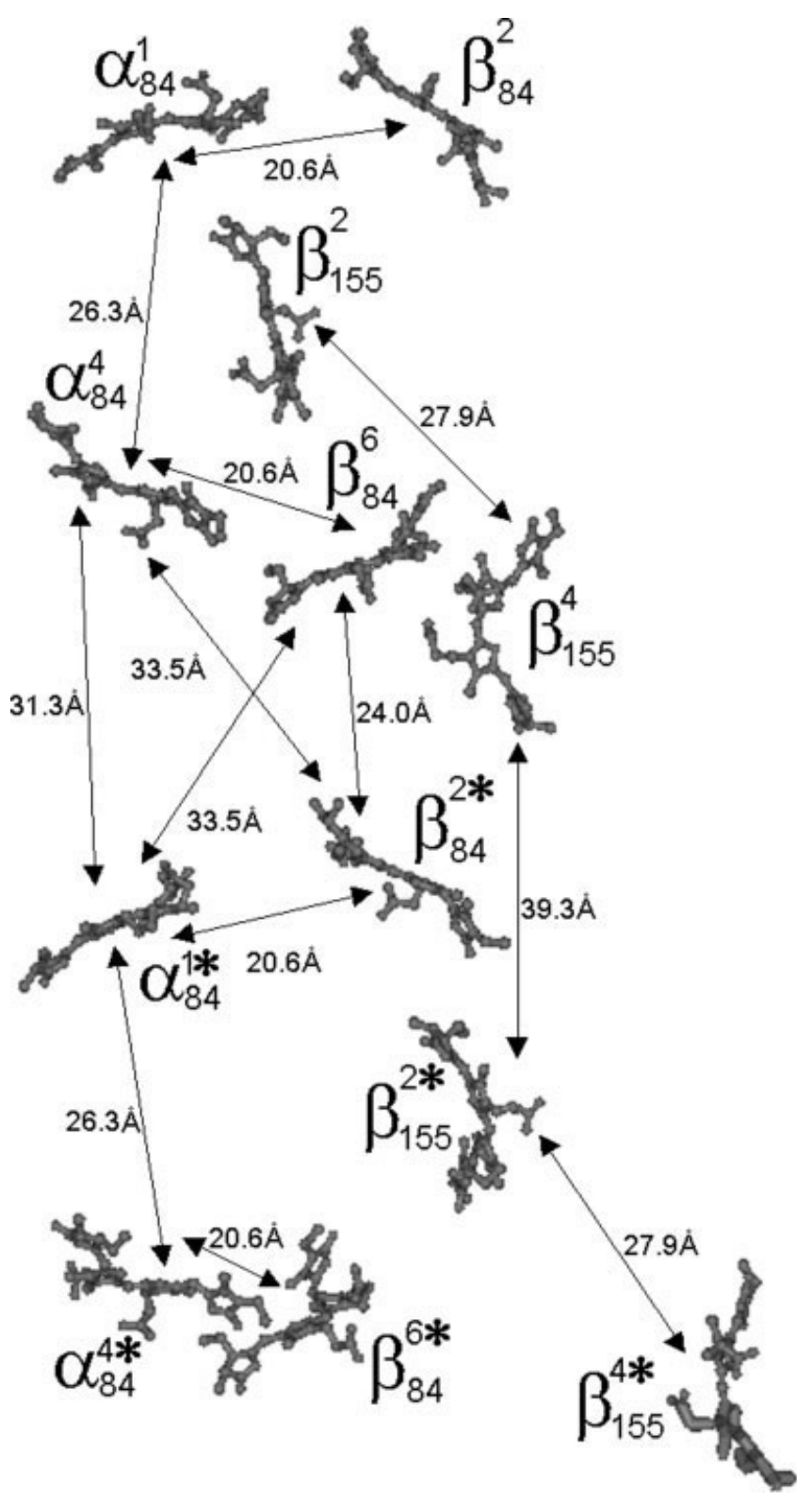

Figure 1. Intra-phycocyanin and inter-phycocyanin preferential light transfer pathways in the rods from Fremyella diplosiphon, showing the distances between the centers of mass of the chromophores. Only the pathways in an $(\alpha \beta)_{2}$ dimer of each hexamer are shown. 
Table 5. Clusters of the Putative Models for a Complex of Two Phycocyanin Hexamers from Fremyella diplosiphon.

\begin{tabular}{rccccc}
\hline $\begin{array}{l}\text { Number } \\
\text { of models }\end{array}$ & $\begin{array}{c}\text { Surface } \\
\text { matching }\left(\AA^{2}\right)\end{array}$ & $\begin{array}{c}\text { Solvation } \\
\text { energy (kcal/mol) }\end{array}$ & $\begin{array}{c}\text { Global } \\
\text { score }\end{array}$ & $\begin{array}{c}\text { Inter-phycocyanin } \\
\text { rotation }\end{array}$ & $\begin{array}{c}\text { Inter-phycocyanin } \\
\text { distance }(\AA)\end{array}$ \\
\hline 3 & 1519 & -10.86 & 0.18 & $9.5^{\circ}$ & 55.4 \\
16 & 1224 & -10.16 & 0.52 & $15.0^{\circ}$ & $5.5^{\circ}$ \\
3 & 1427 & -18.43 & 0.73 & $24.5^{\circ}$ & 55.6 \\
1 & 1792 & -9.75 & 0.00 & $30.0^{\circ}$ & 54.4 \\
6 & 1814 & -13.17 & 0.00 & $35.5^{\circ}$ & 54.6 \\
\hline
\end{tabular}

Positive rotation angles mean rotations of the lower PC anticlockwise around the symmetry axis passing through both centers of the phycocyanin hexamers.

fer among chromophores, the previous calculations and our ones have only considered the aromatic portion of these molecules to calculate the dipole moments. In this work, we have introduced a PM3 semiempirical calculation to determine the dipole moments of the chromophores, whereas Duerring et al. used Schirmer's method of least squares to fit a line to the aromatic portion of the chromophores. Moreover, the previous calculations neglected the spectral differences between the three types of chromophores present in PC. Here, we have used experimentally determined quantum efficiencies, life-times of fluorescence, molar extinction coefficients, and overlap integrals between the emission and absorption fluorescence spectra of the donor and acceptor chromophores for the determination of Förster rate constants. The only other quantum mechanical calculation of dipole moments for chromophores in PBPs reported in the literature is that for phycocyanobilins in PC from Mastigocladus laminosus. ${ }^{35}$ In their calculations, Scharnagl and Schneider used the PPP method and considered the chromophores and their surrounding amino acids as a "super molecule." However, coordinates for the unit cell of PC from Mastigocladus laminosus were never deposited in a public repository and a direct comparison of the result of both calculations protocols is not possible. Nevertheless, Scharnagl and Schneider concluded that the direction of the dipole moments is essentially parallel to the long axis of the chromophores. Such a dipole will be similar to those obtained by the least squares method proposed by Schirmer et al. $^{36}$, and in fact Debreczeny et al. by using both approximations for the dipole moments of the chromophores in PC from Mastigocladus laminosus obtained similar FRET constants. ${ }^{33}$

An experimental transfer time of $1 \mathrm{ps}$ for the pair $\alpha_{84}-\beta_{84}$ in trimers of PC from Synechococcus sp. PCC 7002 has been previously reported. ${ }^{34}$ This transfer time corresponds to transfer constants of 1.42 ps from $\alpha_{84}$ to $\beta_{84}$ and of $3.37 \mathrm{ps}$ from $\beta_{84}$ to $\alpha_{84}$ (experimental transfer times are the reciprocal of the sum of the forward and backward rate constants for a given pair of chromophores). Zhang et al. $^{24}$ have reported experimental transfer times of 10 and 20 ps for chromophore pairs $\alpha_{84}-\alpha_{84}$ and $\beta_{155}-\beta_{155}$, respectively, in hexamers of PC from Anabaena variabilis. These correspond to rate constants of $20 \mathrm{ps}$ for $\alpha_{84}-\alpha_{84}$ and $40 \mathrm{ps}$ for $\beta_{155}-\beta_{155}$. On the basis of the agreement between these experimental results and the calculations presented here, we consider our estimates of Förster constant rates in PC from Fremyella diplosiphon to be an improvement to those previously published. ${ }^{21}$

\section{Docking Model of a Complex of Two Phycocyanins}

PBPs do not exist in isolation but rather form supramolecular complexes called PBSs. To study the assembly of two PC hexamers in the rods of the PBSs from Fremyella diplosiphon, a protein docking was performed using the software BiGGER. From the 5000 putative models of the complex of two PC hexamers generated by BiGGER, only the 20 top-scored solutions for each of the interaction parameters calculated by the software were selected. It is noteworthy that the 20 best evaluated models according to the BiGGER global score showed the expected back-to-back interaction type for PCs in PBSs' rods. ${ }^{11,18,44}$ Conversely, none of the 20 top models for the electrostatic energy parameter showed this type of interaction. Nineteen out of the 20 top scored complexes according to interaction surface, and 11 out of the 20 top scored by solvation energy presented the expected interaction type. Because of the low discriminatory power of the lateral chain interaction parameter (the top 1579 models were assigned the same value), no models using it were selected.

The 50 selected solutions corresponded to 42 different putative models. Solutions were clustered based on inter-phycocyanin rotation angle and distance. Because of the threefold symmetry of the system, models with PCs rotated $120^{\circ}$ around the axial axis were considered equivalent. Also, models where the probe PCs just differed by a few Angstroms of translation along a nonprincipal axis were considered to be equivalent. The resulting six clusters (Table 5) showed inter-phycocyanin distances ranging from 54 to $56 \AA$, and rotation angles ranging from $9.5^{\circ}$ to $35.5^{\circ}$. Where one $\mathrm{PC}$ is upside-down (rotated $180^{\circ}$ around a nonprincipal axis) the existence of a screw angle between the upper and lower trimers in PC hexamers results in models that show angles of rotation different from multiples of the angular step $\left(15^{\circ}\right)$. Therefore, because of the symmetry of the system studied, although we used an angular step of $15^{\circ}$, we have found solutions, which differ from each other by only $5^{\circ}$.

As shown in Table 5, models with an inter-phycocyanin rotation angle of $30^{\circ}$ have the highest surface matching area. This result has been reported by Stec et al. who performed a hexamer contact search for PC from Cyanidium caldarium. ${ }^{23}$ However, the relationship between geometric complementarity and the different energetic contributions to the stabilization of a system are not necessarily trivial as has been shown for trimers of PC from Fremyella diplosiphon. ${ }^{45}$ In fact, Table 5 shows that the 
Table 6. Highest Inter-Phycocyanin Fluorescence Resonance EnergyTransfer Constants at Inter-PC Distance of $55.6 \AA$ and $20.5^{\circ}$ Inter-PC Rotation.

\begin{tabular}{lcccc}
\hline & $\begin{array}{c}\text { Distance } \\
(\AA)\end{array}$ & $\kappa$ & $\begin{array}{c}K_{\mathrm{DA}}^{-1} \\
(\mathrm{ps})\end{array}$ & $\begin{array}{c}K_{\mathrm{AD}}^{-1} \\
(\mathrm{ps})\end{array}$ \\
\hline$\beta_{84}^{4}-\beta_{84}^{3 *}$ & 24.0 & 1.23 & 13.7 & 13.7 \\
$\beta_{84}^{5}-\beta_{84}^{1 *}$ & 24.0 & 1.23 & 13.7 & 13.7 \\
$\beta_{84}^{6}-\beta_{84}^{2 *}$ & 24.0 & 1.22 & 13.8 & 13.8 \\
$\alpha_{84}^{4}-\beta_{84}^{2 *}$ & 33.5 & -1.78 & 38.1 & 58.6 \\
$\alpha_{84}^{5}-\beta_{84}^{3 *}$ & 33.5 & -1.79 & 38.0 & 58.5 \\
$\alpha_{84}^{6}-\beta_{84}^{1 *}$ & 33.5 & -1.79 & 38.0 & 58.6 \\
$\beta_{155}^{4}-\beta_{155}^{2 *}$ & 39.3 & 1.90 & 41.2 & 41.2 \\
$\beta_{155}^{5}-\beta_{155}^{3 *}$ & 39.3 & 1.90 & 41.2 & 41.2 \\
$\beta_{155}^{6}-\beta_{155}^{1 *}$ & 39.3 & 1.90 & 41.2 & 41.2 \\
$\alpha_{84}^{4}-\alpha_{84}^{1 *}$ & 31.3 & 1.24 & 53.5 & 53.5 \\
$\alpha_{84}^{5}-\alpha_{84}^{2 *}$ & 31.3 & 1.24 & 53.5 & 53.5 \\
$\alpha_{84}^{6}-\alpha_{84}^{3 *}$ & 31.3 & 1.24 & 53.4 & 53.4 \\
$\beta_{84}^{4}-\alpha_{84}^{2 *}$ & 33.5 & -1.75 & 60.5 & 39.3 \\
$\beta_{84}^{5}-\alpha_{84}^{3 *}$ & 33.5 & -1.75 & 60.4 & 39.2 \\
$\beta_{84}^{6}-\alpha_{84}^{* *}$ & 33.5 & -1.75 & 60.4 & 39.2 \\
\hline
\end{tabular}

Chromophores on the left belong to the upper phycocyanin hexamer, and the ones on the right (marked with an asterisk) belong to the lower hexamer in the complex.

best scored solutions are those in the cluster with inter-phycocyanin distances of $55.6 \AA$ and a rotation angle of $20.5^{\circ}$. The three solutions in that cluster have the highest solvation energies, and appear in the third, fourth, and sixth positions of the global score. Therefore, these inter-phycocyanin distance and rotation angle were used for the study of the inter-phycocyanin transfer pathways. Similar results (inter-hexamer rotation of $23^{\circ}$ and interphycocyanin distance of $55.1 \AA$ ) have recently been obtained for
PC rods from Gracilaria chilensis by using a different docking algorithm. $^{46}$

\section{Inter-Phycocyanin Preferential Light Transfer Pathways}

We can extend our intra-phycocyanin preferential transfer pathways model to include inter-phycocyanin transfers. On the basis of the above-mentioned docking results, we assume that two neighboring PCs in the rods from Fremyella diplosiphon's PBSs are rotated $20.5^{\circ}$ from each other with a distance between their mass centers of $55.6 \AA$. If we limit our search to transfers between chromophores in the lower trimer of the upper PC, and the upper trimer of the lower PC, 81 paths exist. Similarly to intra-phycocyanin transfers, in the inter-phycocyanin transfers one group is by far faster than the rest. The 15 preferential pathways are shown in Table 6 and Figure 1. The closest chromophores in the complex containing two PC hexamers are those attached to positions $\beta_{84}$. Not surprisingly, $\beta_{84}-\beta_{84}^{*}$ transfer takes on average 13.7 ps (where * means that the chromophore belongs to the lower hexamer of the complex). The next closest pair of chromophores corresponds to those in positions $\alpha_{84}$. However, their interaction is not the second fastest but the fourth with transfer taking on average $53.5 \mathrm{ps}$. Interestingly, the $\beta_{155}-\beta_{155}^{*}$ pairs are the most separated, yet the high orientation coefficient for their dipole moments position them as the third quickest transfer. These cases confirm the importance of not only the distance, but also the orientation factor in the Förster formulation. Transfers from chromophores $\alpha_{84}$ to $\beta{ }_{84}$ and from chromophores $\beta_{84}$ to $\alpha_{84}^{*}$ are also included in the preferential pathways. The six intra-phycocyanin preferential light transfer pathways become 15 inter-phycocyanin preferential light transfer pathways.

Figure 2 shows the $\alpha_{84}-\alpha_{84}^{*}, \alpha_{84}-\beta_{84}^{*}, \beta_{84}-\alpha_{84}^{*}, \beta_{84}-\beta_{84}^{*}$, and $\beta_{155}-\beta_{155}^{*}$ inter-phycocyanin transfer constants between neighboring hexamers as a function of the inter-phycocyanin

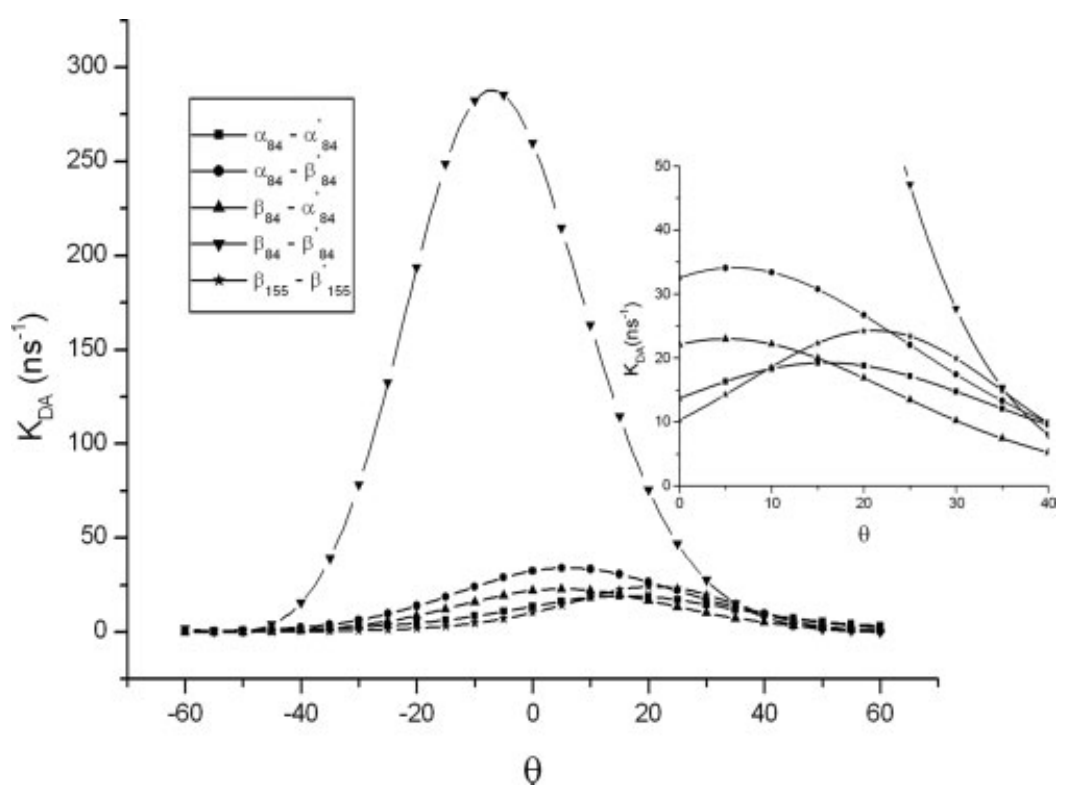

Figure 2. Förster constants for inter-phycocyanin FRET pathways as a function of the inter-phycocyanin rotation angle. An inter-phycocyanin distance of $55.6 \AA$ has been assumed. 
rotation angle. The analysis of the curves reveals that the transfer constant between chromophores $\beta_{84}-\beta_{84}^{*}$ reach a maximum far higher than that on optimally packed rods (inter-phycocyanin rotation of $20.5^{\circ}$ ). The rest of the preferential pathways in the optimally packed rods show transfer constants similar to the maximum obtained in this study. The $\beta_{155}-\beta_{155}^{*}$ pair is the only inter-phycocyanin pathway connecting $\beta_{155}$ chromophores from neighboring hexamers. Remarkably, the transfer between chromophores at positions $\beta_{155}$ is maximized at the angle of rotation predicted by the docking model. This indicates the crucial role of this inter-phycocyanin channel in defining the complete FRET process in Fremyella diplosiphon's PBSs.

Duerring et al. assumed an interhexamer distance of $60 \AA$ and no interhexamer rotation when studying inter-phycocyanin transfer pathways, quoting crystal packing, and electron micrographs as guidelines. ${ }^{21}$ The only strong coupling reported was $\beta_{84}-\beta_{84}^{*}$ with a transfer constant of $46.7 \mathrm{ps}$. In this report, we have been able to identify 15 channels of energy transfer between neighboring PC hexamers. Depending on what chromophore is initially excited, our results show that the energy will be transferred to the neighboring PC hexamer between 13.7 and $85.8 \mathrm{ps}$. These transfer constants are in agreement with experimental transfer times in the range of 45 to 130 ps along PC rods composed of one to four hexamers. ${ }^{47}$

\section{Conclusions}

Using the Förster formulation of FRET and combining the PM3 calculations of the dipole moments of the aromatic portions of the chromophores, docking process via BiGGER software, and experimental data for quantum efficiencies, lifetimes of fluorescence, molar extinction coefficients, and overlap integrals, a semiempirical methodology to model the intra-phycocyanin and the interphycocyanin FRET pathways in PBSs from Fremyella diplosiphon was developed. We identified six intra-phycocyanin and 15 inter-phycocyanin FRET preferential pathways, with transfer constants in agreement with experimental results. It seems that whereas the intra-phycocyanin pathways are optimized for speed, the inter-phycocyanin pathways maximize the number of channels of transference between neighbor hexamers. Our study also predicts the quaternary structure of a rod from Fremyella diplosiphon. The study of the inter-phycocyanin FRET pathways between pairs of chromophores belonging to different PC hexamers as a function of the inter-phycocyanin rotation angle reveals the crucial role of the inter-phycocyanin channel connecting $\beta_{155}$ chromophores to define the complete set of FRET pathways in the system. We conclude that the close agreement between experimental results and our theoretical calculations, as well as the correspondence between the inter-phycocyanin angles of rotation calculated so as to maximize inter-phycocyanin FRET constant and that calculated by protein docking to be the result of the use of PM3 dipole moments for the chromophores in this study.

\section{References}

1. Gantt, E. In Biology of Red Algae; Cole, K. M.; Sheath, R. G., Eds.; Cambridge University Press: New York, 1990, pp. 203-219.
2. Förster, Th. W. Ann Phys (Berlin) 1948, 2, 55.

3. Förster, Th. W. In Modern Quantum Chemistry; Sinanoğlu, O., Ed.; Academic Press: New York, 1965, pp. 93-137.

4. Förster, Th. W. In Comprehensive Biochemistry; Florkin, M.; Stotz, E. H., Eds.; Elsevier: Amsterdam, 1967, pp. 61-77.

5. Agranovich, V. M.; Galanin, M. D. Electronic Excitation Energy Transfer in Condensed Matter; North-Holland: Amsterdam, 1982.

6. Andrews, D. L.; Demidov, A. A. Resonance Energy Transfer; Wiley: Chichester, 1999.

7. Redlinger, T.; Gantt, E. Plant Physiol 1981, 68, 1375.

8. Glazer, A. N. Annu Rev Biophys Biophys Chem 1985, 14, 47.

9. Pullerits, T.; Sundstrom, V. Acc Chem Res 1996, 29, 381.

10. Glazer, A. N. J Biol Chem 1989, 264, 1.

11. Ducret, A.; Sidler, W.; Wehrli, E.; Frank, G.; Zuber, H. Eur J Biochem 1996, 236, 1010.

12. Glazer, A. N. Ann Rev Microbiol 1982, 36, 173.

13. Zolla, L.; Bianchetti, M.; Rinalducci, S. Eur J Biochem 2002, 269, 1534.

14. Ajlani, G.; Vernotte, C. Eur J Biochem 1998, 257, 154.

15. Lundell, D. J.; Glazer, A. N. J Biol Chem 1983, 258, 902.

16. Lundell, D. J.; Glazer, A. N. J Biol Chem 1983, 258, 8708.

17. Yamanaka, G.; Glazer, A. N.; Williams, R. C. J Biol Chem 1980, 255, 11004.

18. Lundell, D. J.; Yamanaka, G.; Glazer, A. N. J Cell Biol 1981, 91, 315.

19. Yu, M. H.; Glazer, A. N.; Williams, R. C. J Biol Chem 1981, 256, 13130.

20. Schirmer, T.; Bode, W.; Huber, R.; Sidler, W.; Zuber, H. J Mol Biol 1985, 184, 257.

21. Duerring, M.; Schmidt, G. B. Huber, R. J Mol Biol 1991, 217, 577.

22. Sauer, K.; Scheer, H.; Sauer, P. Photochem Photobiol 1987, 46, 427.

23. Stec, B.; Troxler, R. F.; Teeter, M. M. Biophys J 1999, 76, 2912.

24. Zhang, J.; Zhao, F.; Zheng, X.; Wang, H. Chem Phys Lett 1999, 304, 357.

25. Demidov, A. A.; Borisov, A. Y. Biophys J 1993, 64, 1375.

26. Pizarro, S. A.; Sauer, K. Photochem Photobiol 2001, 73, 556.

27. Dexter, D. L. J Chem Phys 1953, 21, 836.

28. Jang, S.; Newton, M. D.; Silbey, R. J. Phys Rev Lett 2004, 92, 218301.

29. Mukai, K.; Abe, S.; Sumi, H. J Phys Chem B 1999, 103, 6096.

30. Sumi, H. J Phys Chem B 1999, 103, 252.

31. Scholes, G. D. Annu Rev Phys Chem 2003, 54, 57.

32. Scholes, G. D.; Fleming, G. R. J Phys Chem B 2000, 104, 1854.

33. Debreczeny, M. P.; Sauer, K.; Zhou, J.; Bryant, D. A. J Phys Chem 1995, 99, 8412.

34. Debreczeny, M. P.; Sauer, K.; Zhou, J.; Bryant, D. A. J Phys Chem 1995, 99, 8420.

35. Scharnagl, C.; Schneider, S. J Photochem Photobiol B: Biol 1991, 8, 129.

36. Schirmer, T.; Huber, R.; Schneider, M.; Bode, W.; Miller, M.; Hackert, M. L. J Mol Biol 1986, 188, 651.

37. Stewart, J. J. P. J Comput Chem 1989, 10, 209.

38. Frisch, M. J.; Trucks, G. W.; Schlegel, H. B.; Scuseria, G. E.; Robb, M. A.; Cheeseman, J. R.; Zakrzewski, V. G.; Montgomery, J. A. J.; Stratmann, R. E.; Burant, J. C.; Dapprich, S.; Millam, J. M.; Daniels, A. D.; Kudin, K. N.; Strain, M. C.; Farkas, O.; Tomasi, J.; Barone, V.; Cossi, M.; Cammi, R.; Mennucci, B.; Pomelli, C.; Adams, C.; Clifford, S.; Ochterski, J.; Petersson, G. A.; Ayala, P. Y.; Cui, Q.; Morokuma, K.; Malick, D. K.; Rabuck, A. D.; Raghavachari, K.; Foresman, J. B.; Cioslowski, J.; Ortiz, J. V.; Baboul, A. G.; Stefanov, B. B.; Liu, G.; Liashenko, A.; Piskorz, P.; Komaromi, I.; Gom- 
perts, R.; Martin, R. L.; Fox, D. J.; Keith, T.; Al-Laham, M. A.; Peng, C. Y.; Nanayakkara, A.; Gonzalez, C.; Challacombe, M.; Gill, P. M. W.; Johnson, B.; Chen, W.; Wong, M. W.; Andres, J. L.; Gonzalez, C.; Head-Gordon, M.; Replogle, E. S.; Pople, J. A. Gaussian 98, Revision A. 7, Gaussian, Inc., Pittsburgh, PA, 1998.

39. Schirmer, T.; Bode, W.; Huber, R. J Mol Biol 1987, 196, 677.

40. Grabowski, J.; Gantt, E. Photochem Photobiol 1978, 28, 47.

41. Kleima, F. J.; Hofmann, E.; Gobets, B.; van Stokkum, I. H. M.; van Grondelle, R.; Diederichs, K.; van Amerongen, H. Biophys J 2000, 78,344 .
42. Palma, P. N.; Krippahl, L.; Wampler, J. E.; Moura, J. J. G. Proteins 2000, 39, 372.

43. Krippahl, L.; Moura, J. J.; Palma, P. N. Proteins 2003, 52, 19.

44. Jiang, T.; Zhang, J. P.; Chang, W. R.; Liang, D. C. Biophys J 2001, $81,1171$.

45. Karshikov, A.; Duerring, M.; Huber, R. Protein Eng 1991, 4, 681.

46. Contreras-Martel, C.; Matamala, A.; Bruna, C.; Poo-Caamaño, G.; Almonacid, D.; Figueroa, M.; Martínez-Oyanedel, J.; Bunster, M. Biophys Chem 2007, 125, 388.

47. Holzwarth, A. R. Physiol Plant 1991, 83, 518. 